\title{
Aspartic Acid-derived Wear-preventing and Friction-reducing Agents for Ionic Liquids
}

\author{
Ichiro Minami, ${ }^{* 1}$ Naoko Watanabe, ${ }^{1}$ Hidetaka Nanao, ${ }^{1}$ Sigeyuki Mori, ${ }^{1}$ Kenta Fukumoto, ${ }^{2}$ and Hiroyuki Ohno ${ }^{2}$ \\ ${ }^{1}$ Iwate University, 4-3-5 Ueda, Morioka 020-8551 \\ ${ }^{2}$ Tokyo University of Agriculture and Technology, 2-24-16 Nakamachi, Koganei, Tokyo 184-8588
}

(Received December 14, 2007; CL-071387; E-mail: ichiro@iwate-u.ac.jp)

The first additives that improve the tribological properties of ionic liquids were designed. They were evaluated by a ballon-flat type tribo-test under reciprocating motion. Tetraalkylammonium and tetraalkylphosphonium salts of N-protected aspartic acid was dissolved in 1-alkyl-3-methylimidzolium bis(trifluoromethylsulfonyl)imide. They prevented wear remarkably and reduced friction considerably. The salt of N-protected glutamic acid prevented wear but did not reduce friction under the conditions we have evaluated. A new model of boundary film composed of liquid clathrate structure is proposed herein.

Ionic liquids are unique fluids that possess non-flammability, non-volatility, and outstanding thermo-oxidative stability. These properties are desirable for lubricating fluids, especially for lubricants used under extreme conditions. Since ionic liquids are composed of anion and cation, variation in their structure is estimated more than one million compounds. ${ }^{1}$ The tribological properties of certain ionic liquids have been reported. ${ }^{2}$ In our previous work, 1,3-dialkylimidazolium-derived ionic liquids were found to exhibit comparable tribological properties to the conventional synthetic lubricants such as perfluoropolyethers. ${ }^{3}$ We also pointed out the importance of additives that improve the tribological properties of ionic liquids. However, ionic liquids are strange solvents; they hardly dissolve the conventional lubricant additives. Simple carboxylic acids improved the tribological properties of dialkylimidazorium-derived ionic liquids. ${ }^{4}$ However, these carboxylic acids are volatile and corrosive. Based on our concept of molecular design of lubricant additives, ${ }^{5}$ chemical modification of carboxylic acid by introduction of certain functional group was examined. Here, we wish to report molecular design of the first additives that improve anti-wear properties of ionic liquids.

In our preliminary work, we found that dicarboxylic acids such as succinic and glutaric acid reduced friction of 1butyl-3-methylimidazolium bis(trifluoromethanesulfonyl)imide (BIMI-TFSI), while adipic acid did not. Solubility of these simple acids in BMIM-TFSI still remains the problem. These simple compounds are volatile and corrosive in fact, however the relation between chemical structure and the tribological properties are of interest. Recently, aspartic acid- and glutamic acid-derived ionic liquids were developed. ${ }^{6-8}$ The structural features of them are functionalized dicarboxylic acid obtained from natural resources. The ionic structure is beneficial to dissolve them into ionic liquids. Thus, we synthesized trialkylammonium and trialkylphosphonium salts of these amino acids. The free amino group was protected by benzyl or acetyl group to improve the stability. Structure of the designed additives is shown in Figure 1. Samples for the tribo-test were prepared by dissolving the additive in BIMI-TFSI at the concentration of $10 \mathrm{mmol} \mathrm{kg}^{-1}$.

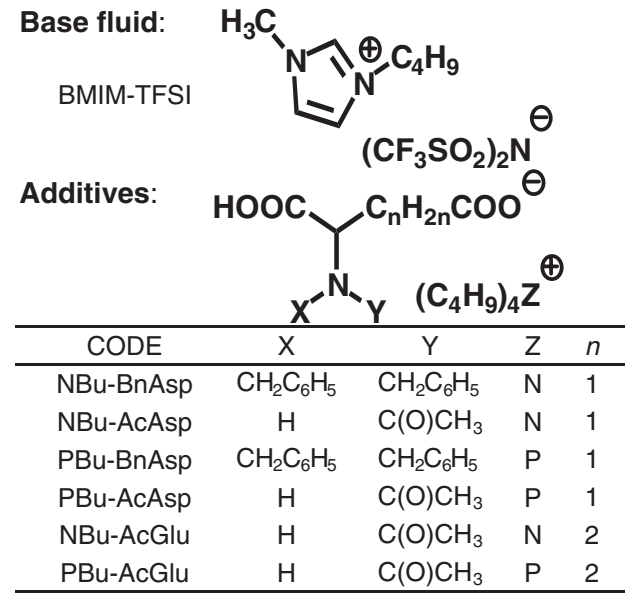

Figure 1. Structure of the base fluid and the additives.

The tribological properties were obtained using a ball-onflat type tribo-tester under reciprocating motion at $75^{\circ} \mathrm{C}$ for $30 \mathrm{~min}$. Test conditions are; applied load of $20 \mathrm{~N}$ (corresponds to Hertz contact stress of $1.7 \mathrm{GPa}$ ), frequency of $1.0 \mathrm{~Hz}$, amplitude of $5.0 \mathrm{~mm}$. The ball specimen was $6.35 \mathrm{~mm}$ in diameter made of SUJ2 steel (JIS). The flat specimen was $\Phi 25 \times 7 \mathrm{~mm}$ made of SUJ2 steel (JIS). Friction force was monitored throughout the test. The data was converted to friction coefficient. Width of wear scar on ball and flat specimen after the test were measured by an optical microscope. Two tests were run for each set of test parameters, and differences in the results were less than $15 \%$.

Friction trace during the tribo-test is summarized in Figures 2 and 3. Low friction without fluctuation is desirable as lubricants. Friction of the additive-free BMIM-TFSI gradually increased after running-in period for $250 \mathrm{~s}$. Fluctuation of friction was also observed throughout the friction test. $\mathrm{NBu}-\mathrm{AcGlu}$ and $\mathrm{NBu}-\mathrm{AcAsp}$ showed similar friction trace after a short running-in period. It should be noted that $\mathrm{NBu}-\mathrm{BnAsp}$ provided low friction throughout the test. Stable friction without fluctuation was observed at the test duration after $1000 \mathrm{~s}$. The phosphonium salts showed the results similar to the ammonium salts. Among them, $\mathrm{PBu}-\mathrm{BnAsp}$ provided low friction under these conditions. The results indicate that the anionic moiety, BnAsp plays an important role on reducing friction.

Wear-width on ball and on flat after the tribo-test was measured by an optical microscope. Average of the width on ball and flat is summarized in Figure 4. All additives prevented wear. Phosphonium salts are better than ammonium salts, except for $\mathrm{NBu}-\mathrm{BnAsp}$. A possible mechanism for the wear prevention by phosphonium salts is the formation of certain protecting film composed of phosphate on rubbed surfaces. ${ }^{9}$

Besides the film forming from phosphonium moiety, $\mathrm{N}$ - 


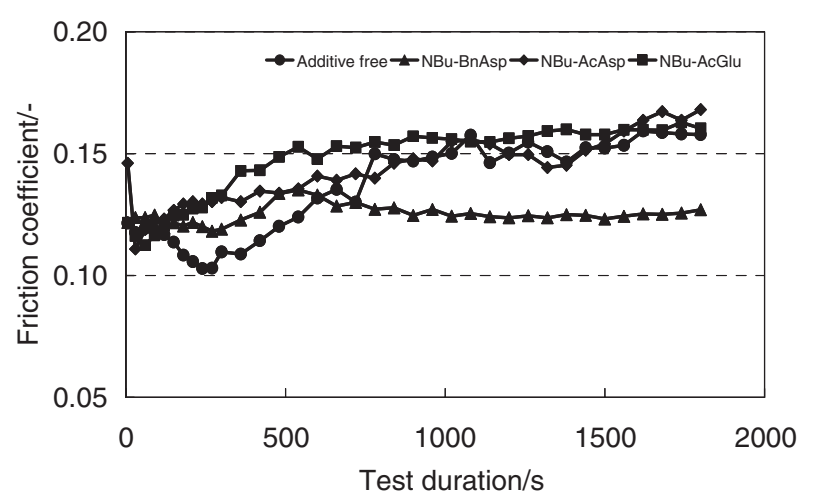

Figure 2. Friction trace of ammonium salts in BIMI-TFSI by a ball-on-flat type tribo-test at $20 \mathrm{~N}$.

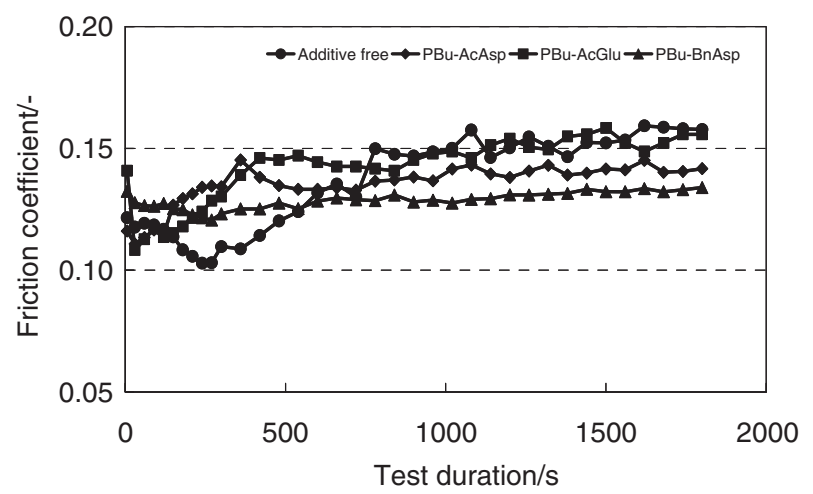

Figure 3. Friction trace of phosphonium salts in BIMI-TFSI by a ball-on-flat type tribo-test at $20 \mathrm{~N}$ for $30 \mathrm{~min}$.

benzyl-protected aspartic acid moiety seems to play significant roles in improving the tribological properties. Adsorption of carboxylic acid on rubbing steel surfaces is known to reduce friction. ${ }^{10}$ The di-acid moiety is beneficial for strong affinity with steel surface. ${ }^{5}$ However, the role of the remaining parts of the molecule, $\mathrm{N}$-protected amine, on wear preventing cannot be explained by the classical boundary lubrication mechanism. ${ }^{10}$ All the additives provided smooth worn surface by the rubbing process, as shown in the "Graphical Abstract". This suggests a formation of boundary film during the rubbing process. One possible model is formation of a liquid clathrate from the additive and the base fluid. The first step should be the adsorption of carboxyl group on steel surface. Since the additive is composed of anionic and cationic moieties, the Coulomb force between the adsorbed molecule and the base fluid (BMIM-TFSI) is possible. This results in an interfacial layer on the rubbing surface that is composed of anchor moiety (the additive) and mobile phase

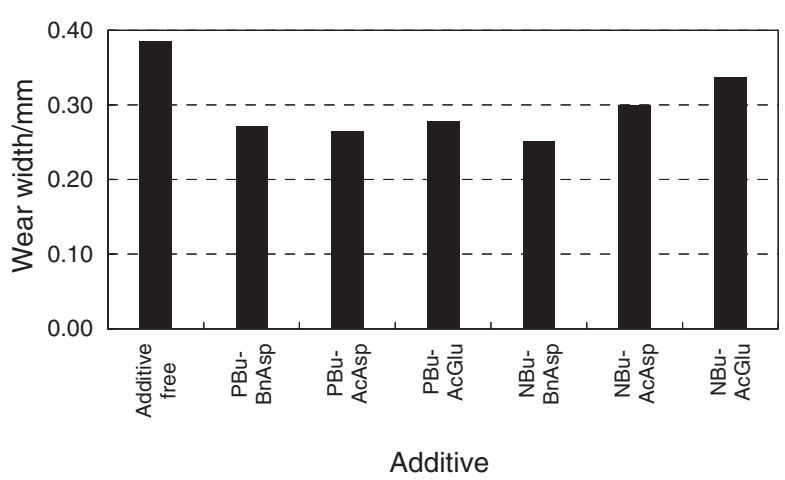

Figure 4. Wear width after a ball-on-flat type tribo-test at $20 \mathrm{~N}$.

(BMIM-TFSI). This interfacial layer prevents direct metalmetal contact during the friction test; thereby preventing wear. The presence of the mobile phase is beneficial to decrease the shear stress; thereby reducing friction. The role of phenyl moiety in the N-protective group would be enforcement of the aromatic interaction with imidazolium moieties in BMIM-TFSI. ${ }^{11}$ Here, we propose that lubricity of ionic liquids can be improved by appropriate additive technology. The mechanism of lubrication by the additives is now under investigation.

This research was supported by the Grant-in-Aid for Scientific Research on Priority Areas, "Science of Ionic Liquids" from the Ministry of Education, Culture, Sports, Science and Technology of the Japanese Government.

\section{References}

1 R. A. Reich, P. A. Stewart, J. Bohaychick, J. A. Urbanski, Lubr. Eng. 2003, 59, 16.

2 C. Ye, W. Liu, Y. Chen, L. Yu, Chem. Commun. 2001, 2244.

3 H. Kamimura, T. Kubo, I. Minami, S. Mori, Tribol. Internat. 2007, 40, 620 .

4 H. Kamimura, T. Chiba, N. Watanabe, T. Kubo, I. Minami, S. Mori, Tribol. Online 2006, $1,40$.

5 I. Minami, S. Mori, Lubr. Sci. 2007, 19, 127.

6 K. Fukumoto, M. Yoshizawa, H. Ohno, J. Am. Chem. Soc. 2005, 127, 2398.

7 K. Fukumoto, H. Ohno, Chem. Commun. 2006, 3081.

8 J. Kagimoto, K. Fukumoto, H. Ohno, Chem. Commun. 2006, 2254.

9 E. S. Forbes, J. Battersby, ASLE Trans. 1974, 17, 263.

10 F. P. Bowden, D. Tabor, The Friction and Lubrication of Solids, 200 Oxford University Press, 2001.

11 J. D. Holbrey, W. M. Reichert, M. Nieuwenhuyzen, O. Sheppard, C. Hardacre, R. D. Rogers, Chem. Commun. 2003, 476 . 